\title{
Des crues et des échelles
}

\author{
About floods and scales
}

par Pierre Hubert

UMR Sisyphe, CIG, Ecole des Mines de Paris, Fontainebleau

Multifractal approaches are now used in hydrology scale changes and should lead to a rational base for this type of analyses. It was displayed that hydrometeorological phenomena have some scale-invariant characteristics, so that normal and extreme events should be considered in the same conceptual frame. The practical consequences are important because it can be assigned to events considered as exceptional a much lower return-time than with classical approaches.

\section{I $\square$ INTRODUCTION}

Nous aborderons dans cet article des notions qui ne sont peut-être pas familières à la majorité des lecteurs, celle d'invariance d'échelle en particulier. Nous proposons aussi une démarche inductive dont le moins qu'on puisse dire est qu'elle est peu usitée en hydrologie. Nous voudrions cependant souligner d'emblée que ce langage et cette démarche ne sont pas de simples coquetteries de chercheur, mais qu'ils visent à interpréter des observations, à formaliser des connaissances et, au-delà, à améliorer les outils que l'hydrologie en tant que science se doit de fournir à ceux qui l'utilisent au quotidien pour résoudre des problèmes.

Il est devenu banal de parler de l'extrême variabilité des phénomènes hydrométéorologiques. Si ce n'est certainement pas d'hier que les hommes ont remarqué les caprices du temps et les débordements des cours d'eau, le développement des moyens d'observation et de mesure d'une part, une plus grande vulnérabilité des activités humaines au risque hydrologique et une volonté affirmée de le maîtriser d'autre part, leur ont permis de mieux appréhender cette variabilité. Il est apparu que cette variabilité était fonction de l'échelle d'observation et de représentation. Nous voudrions en donner deux exemples très empiriques issus de l'étude des précipitations.

Le premier concerne la carte des isohyètes de l'année 1986 sur le territoire du Burkina Faso (figure 1). A partir des 113 postes pluviométriques du réseau national, on a pu tracer, manuellement, la carte nationale de la figure 1. Or pendant l'année 1986 le réseau de recherche EPSAT86 [1] comportant 120 stations, incluant une dizaine de stations du réseau national, a fonctionné dans le degré-carré (d'environ
$100 \mathrm{~km}$ de côté) entourant la ville de Ouagadougou. Ces données supplémentaires permettent de tracer une nouvelle carte à l'échelle du degré-carré qui présente à cette échelle plus fine une variabilité à laquelle on ne s'attendait pas.

La variabilité spatiale des précipitations, que l'on aurait pu penser voir s'apaiser, surtout à l'échelle annuelle, continue de se manifester avec beaucoup de vivacité à l'échelle kilométrique. Le radar météorologique a permis de faire des observations analogues, mettant en évidence une extrême

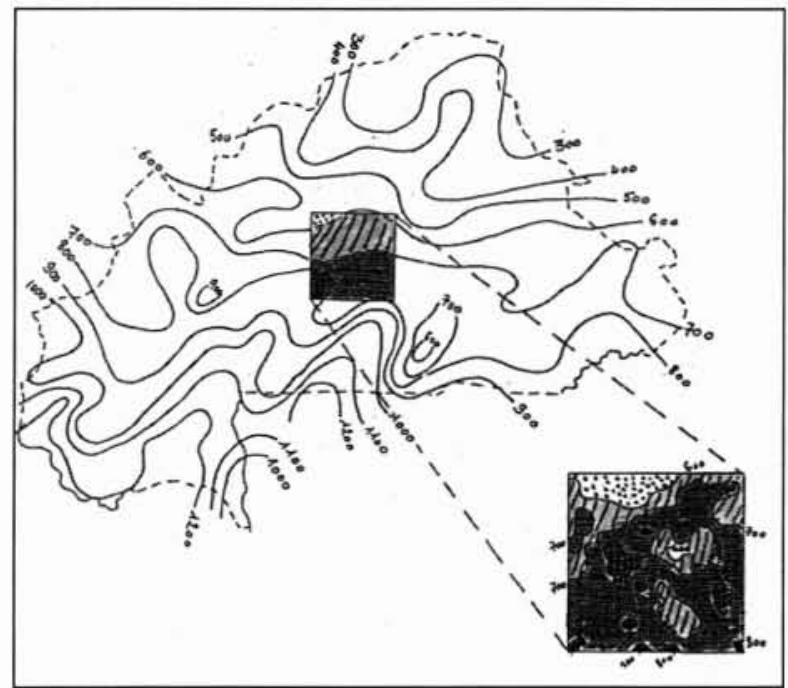

1. Isohyètes du Burkina Faso pour l'année 1986, à l'échelle nationale (113 postes) et à l'échelle du degré-carré de Ouagadougou (120 postes dont une dizaine du réseau national). 


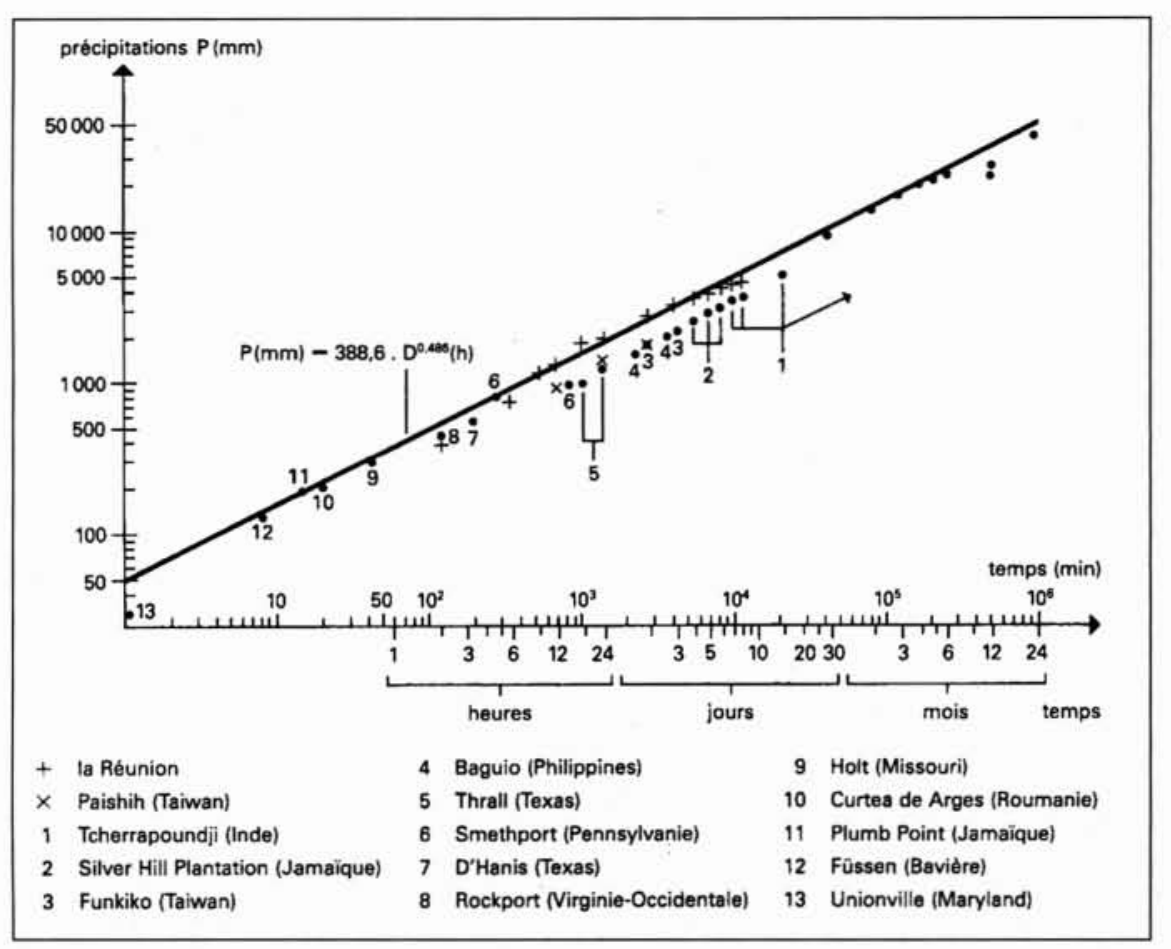

2. Valeurs maximales de précipitations observées pour différents intervalles de temps.

variabilité spatiale qui met à mal certaines hypothèses de la théorie de I'hydrogramme unitaire et qui rend délicate, surtout pour les petits pas de temps, l'utilisation de techniques telles que la géostatistique [2,3] pour la modéliser. Est-ce à dire que cette variabilité n'est pas maîtrisable, même au plan mathématique ? L'exemple suivant qui concerne les records de précipitations observées pour diverses échelles de temps (figure 2) devrait nous donner des raisons d'espérer !

Cette figure a été publiée dans de nombreux ouvrages et articles d'hydrologie $[4,5]$. Elle montre que les maximums enregistrés dépendent de la durée d'observation, ce qui n'a rien d'étonnant, mais que cette dépendance n'est pas linéaire et donc que la pluie n'est pas homogène au cours du temps. Il est cependant remarquable qu'une organisation de ces valeurs apparaisse sur un diagramme $\log -\log$ et qu'il existe un lien entre la statistique empirique (le maximum observé) et l'échelle de temps correspondante. Ce lien, et les paramètres qui le caractérise, sont un exemple ici très empirique d'invariance d'échelle dont des interprétations plus théoriques ont pu être proposées [6]. C'est un lien de cette nature que Francou et Rodier [7] ont noté, pour les crues maximales observées dans le monde, les observations empiriques étant organisées selon une échelle spatiale.

\section{II — UN PEU DE THÉORIE}

C'est en ayant explicitement en tête ce problème de la dépendance de certaines mesures à l'échelle d'observation que Mandelbrot $[8,9]$ a créé la géométrie fractale, en s'appuyant sur des résultats mathématiques oubliés ou occultés du début du siècle, qu'il a développés et appliqués à de nombreux problèmes des sciences de la nature. Il a reconnu dans une dimension fractale (non-entière) le lien susceptible de relier une mesure et une échelle de mesure pour de nombreux objets géométriques susceptibles de modéliser des objets naturels. On doit noter certaines applications hydrolo- giques de cette géométrie, en particulier pour la description des réseaux hydrographiques et des bassins versants, mais aussi pour caractériser le support temporel des précipitations $[10,11]$.

Ces résultats ne doivent cependant pas nous faire oublier que des phénomènes complexes tels que la précipitation, et plus encore l'écoulement, ne se résument pas à occurrence ou non occurrence et qu'il convient de se préoccuper de l'intensité de la pluie ou de l'écoulement selon l'échelle de temps considérée. La notion d'intensité est d'ailleurs implicitement présente dans la définition du seuil de référence définissant un objet géométrique, par exemple l'occurrence de pluie, et on a pu remarquer [12] que la dimension fractale de l'occurrence de pluie est une fonction décroissante du seuil de référence. Cette dépendance de la dimension d'un ensemble à la valeur de son seuil de référence, déjà notée par Schertzer et Lovejoy [13] ou par Halsey et al [14], amène à dépasser en pratique dans ce type d'études la notion d'objet fractal au profit de celle de champ multifractal.

L'approche multifractale vise à relier échelle et intensité pour des processus en cascade concentrant la matière et/ou l'énergie dans des domaines spatio-temporels de plus en plus ténus $[15,16]$. Les modèles multifractals auxquels nous nous intéresserons ont d'abord été développés comme modèles phénoménologiques de la turbulence. Ils ont été conçus pour reproduire dans des cascades multiplicatives les principales propriétés (symétrie, conservation,...) des équations non linéaires (Navier et Stokes) qui régissent la dynamique de ce phénomène. Ces modèles de cascades devraient être utiles pour l'étude de l'atmosphère et de la pluie car, même si les équations aux dérivées partielles (inconnues) gouvernant l'atmosphère et la production de pluie sont certainement plus complexes que celles de la turbulence hydrodynamique, ces équations partagent vraisemblablement leurs propriétés fondamentales. Nous pensons qu'il devrait en être de même pour les débits qui résultent d'une intégration complexe et non 
conservative, dans l'espace et dans le temps, des précipitations. La similarité des comportements asymptotiques des distributions statistiques des pluies et des débits a d'ailleurs été déjà postulée [17] et a fait l'objet de développements opérationnels.

Considérer un champ spatial et/ou temporel comme multifractal revient à le caractériser à la fois de façon multiéchelle et multi-intensité : les intensités de plus en plus fortes du champ correspondent à des singularités de plus en plus extrêmes et de plus en plus rares, donc associées à des dimensions fractales de plus en plus faibles. Contrairement à la plupart des modèles, toutes les singularités, moyennes comme extrêmes, sont générées par le même processus élémentaire. Le théoricien n'a plus à ajouter à la main les anomalies (les "horsains" selon l'heureux emprunt de J.M. Masson) puisqu'elles existent en germe dans le champ moyen et l'expérimentateur n'a plus à discriminer laborieusement le comportement extrême du comportement ordinaire.

Ce lien a priori insolite entre extrêmes et moyenne d'un champ peut être d'abord compris par des propriétés d'universalité : bien qu'un champ multifractal dépende d'une infinité de paramètres, seuls un petit nombre d'entre eux peuvent finalement se révéler pertinents, les autres étant en quelque sorte lessivés par la répétition du processus élémentaire. L'exemple classique d'universalité est le mouvement brownien, attracteur universel de toute marche aléatoire dont la variance des pas infinitésimaux est finie. Dans le cas des processus multifractals, bien que nous ayons plutôt à considérer des processus multiplicatifs, des propriétés d'universalité similaires ont été mises en évidence et c'est l'index de multifractalité alpha (compris entre 0 et 2), proportionnel au rayon de courbure de la codimension des singularités autour du champ moyen, qui détermine la distribution des extrêmes [18].

Ce lien direct entre champ moyen et extrêmes peut aussi être relié à un phénomène physique, connu sous l'appellation d'auto-organisation critique, dès que l'on considère le comportement du champ de pluie sur un grand nombre d'échantillons. En effet, à partir d'une certaine singularité critique $q_{D}$. l'intensité observée du champ de précipitation est fréquemment bien plus importante que celle prévue par un modèle théorique ne prenant en compte que les échelles supérieures à l'échelle d'observation. Cela est dû au fait que non seulement les fluctuations à petite échelle restent perceptibles à grande échelle mais que finalement elles pilotent les extrêmes à cette échelle. Ce lien entre microscopique et macroscopique est analogue aux transitions de phase des systèmes conservatifs où la distance de corrélation diverge pour une température critique ; ici, c'est le rapport effectif d'échelle qui se met à diverger. Parmi les multiples implications de cette "transition de phase multifractale de premier ordre" il faut noter une chute algébrique (c'est-à-dire lente) de la distribution de probabilité des intensités au-delà d'un certain niveau :

$$
\text { Prob }[X>x] \approx x^{q D}
$$

Il est important de noter que cette chute algébrique de probabilité entraîne la divergence des moments statistiques d'ordre supérieur à $q_{D}$, divergence qui a de nombreuses conséquences théoriques et expérimentales puisque la loi des grands nombres ne s'applique plus, d'où perte d'ergodicité, divergence des estimateurs usuels, sensibilité des estimations à la taille des échantillons, etc. Les conséquences pratiques d'un tel comportement algébrique de la distribution de probabilité sont considérables, car les lois algébriques décroissent infiniment plus lentement que les lois à caractère exponentiel habituellement utilisées pour la prédétermination d'événements de récurrence donnée, qui seraient alors considérablement sous-estimés.

\section{III $\square$ APPLICATIONS AUX SÉRIES ET CHAMPS HYDROMÉTÉOROLO- GIQUES}

\subsection{Applications aux précipitations}

L'approche multifractale a connu de nombreuses applications dans l'étude et la modélisation des séries pluviométriques et des champs précipitants $[19,20]$. Nous citerons en particulier une note sur la série pluviométrique de Nîmes [21] et une étude concernant les longues séries pluviométriques recueillies dans le cadre du projet FRIEND-AMHY [22]. Les résultats de ces analyses sont encourageants quant aux possibilités d'une modélisation multifractale des séries pluviométriques et les estimations des paramètres de ces modèles sont souvent concordantes. Les divers auteurs montrent que les séries pluviométriques présentent un caractère scalant sur de grandes gammes d'échelles, allant de quelques heures à plusieurs mois, voire plusieurs années. Un changement de régime aux alentours de la semaine (maximum synoptique) a parfois été signalé. Parmi les paramètres des relations multifractales, le moment critique $q_{D}$ est particulièrement intéressant, puisqu'il détermine la limite de divergence des moments. Les estimations issues de l'étude de différentes stations concordent, ce qui tendrait à suggérer que l'on tient là une caractéristique intrinsèque des pluies. Leurs valeurs sont légèrement supérieures à 3 . Pratiquement cela signifie qu'il serait vain de chercher à estimer un coefficient d'aplatissement (faisant intervenir le moment d'ordre 4) et que les estimations du moment d'ordre 3 (coefficient d'asymétrie) risquent de ne converger qu'avec une extrême lenteur. Cette dernière constatation conforterait la méfiance que de nombreux praticiens manifestent à l'égard de cette statistique et des lois qui l'utilisent.

Au-delà d'études particulières, l'approche multifractale a permis de proposer une modélisation des courbes intensitédurée-fréquence [23], ouvrant la voie à une modélisation des régimes. Les paramètres caractérisant les courbes intensitédurée-fréquence semblent en effet pouvoir être déduits des paramètres multifractals des séries chronologiques correspondantes. Enfin une avancée décisive a été réalisée par l'intégration effective des aspects spatiaux et temporels [24], dont les applications au niveau de la prévision devraient être considérables.

\subsection{Applications aux séries de débits}

L'application des concepts d'invariance d'échelle aux débits des rivières est à la fois plus récente et par voie de conséquence plus limitée. Il faut toutefois noter qu'elle se situe dans la lignée des travaux de Hurst [25] qui, le premier, avait mis en évidence, à partir de préoccupations fort pratiques, 
des dépendances statistique à long terme dans les séries chronologiques de débits. Turcotte et Greene [26] ont étudié la fréquence des crues de 10 rivières américaines. Ils caractérisent l'invariance d'échelle qu'ils mettent en évidence pour des échelles de temps allant de 1 à 100 ans, par le rapport de la crue centennale à la crue décennale, égal dans le cadre de cette approche au rapport de la crue décennale à la crue annuelle. Ce rapport varie de 2 à 8 environ et les auteurs associent ces variations aux différences climatiques des bassins considérés. Tessier et al [27] ont étudié les séries de pluies et de débits de 30 bassins français dont la surface allait de 40 à $200 \mathrm{~km}^{2}$. Ils mettent en évidence une invariance d'échelle pour des durées allant de la journée à trente ans, et notent un changement de régime autour de 16 jours qu'ils attribuent au maximum synoptique. Le paramètre $q_{D}$ (moment critique de divergence) estimé à partir de l'ensemble des données est de l'ordre de 3,2 pour les échelles de temps supérieures à 30 jours, de l'ordre de 2,7 pour les échelles de temps inférieures à 16 jours (avec une plage d'erreur très importante). Une étude plus récente de Pandey et al [28] s'est intéressée à 19 bassins américains dont la taille va de $5 \mathrm{~km}^{2}$ à près de 2 millions de $\mathrm{km}^{2}$ (ce dernier bassin étant celui du Mississipi) totalisant près de 700 années-stations. Ils concluent à un comportement multifractal pour des échelles de temps allant de 23 à 216 jours. Ils remarquent également un changement de régime aux alentours de 8 jours. En ce qui concerne les paramètres multifractals, en particulier le paramètre $q_{D}$ qu'ils estiment en moyenne à 3,1 , leurs estimations se rapprochent de celles de Tessier [27] mais, contrairement à Turcotte [26] ils attribuent au seul hasard la dispersion des estimations relatives aux différents bassins.

\section{IV — CONCLUSIONS ET PERSPECTIVES}

Après cette brève présentation de l'approche multifractale et de quelques-unes de ses applications en hydrologie, nous voudrions surtout tracer quelques perspectives. Nous partons d'un modèle phénoménologique de cascade multiplicative issu d'études sur la turbulence hydrodynamique, conçu pour reproduire les symétries de l'équation de Navier et Stokes. Même si nous ne connaissons pas les équations aux dérivées partielles gouvernant la formation des pluies et des débits, il est raisonnable de penser qu'elles partagent certaines caractéristiques de l'équation de Navier et Stokes. Cette conjecture donne une certaine base physique à l'approche multifractale des pluies et des débits. Les diverses applications réalisées à ce jour sont encourageantes, même si l'estimation des paramètres de ces modèles reste encore trop imprécise. Un comportement invariant d'échelle pour des durées allant grossièrement de la journée au siècle peut être mis en évidence. Cette invariance d'échelle devrait à terme permettre de surmonter les approches trop parcellaires ou locales, difficiles ou impossibles à généraliser, qui sont de mise aujourd'hui et qui ont en particulier conduit, dans le domaine de l'estimation des crues en particulier, à un foisonnement incontrôlable de lois statistiques ad hoc, quand ce n'est pas à des attitudes autoritaires et bureaucratiques [29] comme c'est le cas aux Etats-Unis où c'est la loi (civile) qui "impose" aux crues d'obéir à la loi (statistique) log-Pearson III. Le point qui nous semble le plus essentiel est que l'approche multifractale sug- gère que la décroissance des lois de probabilité des variables hydrologiques serait de nature algébrique (décroissance en $x^{q D}$ ) et non exponentielle (décroissance en $\mathrm{e}^{-a r}$ ) comme c'est peu ou prou le cas de toutes les lois utilisées actuellement. Soulignons encore une fois l'importance pratique de ce point car le débit correspondant à un durée de retour donnée estimé à partir de l'hypothèse algébrique risque d'être beaucoup plus important que celui estimé à partir de I'hypothèse exponentielle. Dans cette dernière hypothèse, on passe du débit de durée de retour $N$ années au débit de durée de retour $10 * N$ par l'addition d'une constante. Dans l'hypothèse algébrique, c'est une multiplication par une constante qui permet de passer du débit de durée de retour $N$ années au débit de durée de retour $10 * N$. Il s'agit donc d'éclairer ce choix fondamental à côté duquel certaines querelles agitant le monde de la statistique hydrologique apparaissent futiles. Face à l'importance des enjeux théoriques et pratiques, il est donc nécessaire de poursuivre les investigations concernant l'approche multifractale des pluies et des débits, pour en éclairer les fondements au plan théorique et pour en valider définitivement la pertinence au plan de l'analyse des données.

\section{REMERCIEMENTS}

Ce travail a été réalisé dans le cadre du Programme National de Recherche en Hydrologie de I'INSU (Projet 99 PNRH 27).

\section{BIBLIOGRAPHIE}

[1] Hubert, P., CARBonNel, J.P. (1988) Caractérisation fractale de la variabilité et de l'anisotropie des précipitations intertropicales. CRAS. Série II, 307, 909-914.

[2] Matheron, G. (1965) Les variables régionalisées et leur estimation, Masson, Paris,

[3] De.номме, J.P. (1978) Applications de la théorie des variables régionalisées dans les sciences de l'eau, Bull. du BRGM, section III, 4, 341-375

[4] Raudkivi, A.J. (1979) Hydrology, Pergamon Press, New York.

[5] Réménieras, G., Hubert, P. (1990) Hydrologie, Encyclopaedia Universalis, volume $\mathrm{XI}, 796-806$.

[6] Hubert, P., Tessier, Y., Lovejoy, S., SCHERIZER, D., SChMITT, F., Ladoy, P., Carbonnel, J.P., Violette, S., Desurosne, I. (1993) Multifractals and extreme rainfall events, Geophysical Research Letters, 20, 931-934.

[7] FrancoU, J., Rodier, J.A. (1967) Essai de classification des crues maximales observées dans le monde, Cah. O.R.S.T.O.M. sér. Hydrol., IV, 3 , 19-26.

[8] MandelBRot, B.B. (1975) Les objets fractals, forme, hasard et dimension, Flammarion, Paris, $192 \mathrm{p}$.

[9] MANDel.brot, B.B. (1977) The fractal geometry of nature, Freeman, San-Francisco, $461 \mathrm{p}$.

[10] Hubert, P., CARBonNEl, J.P. (1989) Dimensions fractales de l'occurrence de pluie en climat soudano-sahélien, Hydrologie Continentale, 4, 3-10.

[11] Lovejoy, S., SCHERTZFr, D., Tsonis, A.A. (1987) Functional box-counting and multiple elliptical dimensions in rain, Science, 235, 1036-1038.

[12] Hubert, P., Friggitt, F., CARbonnel, J.P. (1995) Multifractal structure of Rainfall Occurrence in West Africa, New Uncertainty Concepts in Hydrology and Water Resources, Z.W. Kundzewicz editor, Cambridge University Press, 109-113.

[13] SCHERTzer, D., Lovejoy, S. (1984) On the dimension of atmospheric motion, Turbulence and chaotic phenomena in fluids, T. Tatsumi editor. North Holland, 505-508. 
[14] Halsey, T.C. Jensen, M.H., Kadanoff, L.P., Procaccia, I., Shraiman B.I. (1986) Scaling measures and singularities, Phys. Rev. A, 33, 1141.

[15] LOVEJOY, S., SCHERTZER, D. (1986) Scale invariance, symmetries, fractals and stochastic simulations of atmospheric phenomena, Bulletin of the AMS, 67, pp 21-32.

[16] SCHERIZER, D., Lovejoy, S. (1987) Physical modeling and analysis of rain and clouds by anisotropic scaling and multiplicative processes, J. Geophys. Res., 92, D8, pp 9693-9714.

[17] Gunlot, P., DuBAND, D. (1967) La méthode du GRADEX pour le calcul de la probabilité des crues à partir des pluies, Publication AIHS $n^{\circ} 84$. 560-569.

[18] SCHERTZER, D., LovEJoY, S. (1991 Scaling nonlinear variability in Geodynamics : Multiple singularities, observables, universality classes, Nonlinear variability in Geophysics, Scaling and Fractals, D. Schertzer and S. Lovejoy editors, Kluwer, 41-82.

[19] OLsson, J. (1996) Validity and applicability of scale-independant, multifractal relationship for rainfall. Journal of atmospheric research, 42, 5365.

[20] DE LIMA, M.I.P. (1998) Multifractals and the temporal structure of rainfall, Doctoral dissertation, Wageningen Agricultural University, 222 p.

[21] Ladoy, P., Schmitt, F., Scherizer, D., Lovejoy, S. (1993) Analyse multifractale de la variabilité pluviométrique à Nîmes, CRAS, Paris, Série II, 317, 775-782.
[22] BeNDJOUDI, H., HuBERT, P. (1998) A propos de la distribution statistique des cumuls pluviométriques annuels. Faut-il en finir avec la normalité ? Revue des Sciences de l'Eau 4, 617-630.

[23] Bendjoudi, H.. Hubert, P., SChertztr, D., Lovejoy, S. (1997) Interprétation multifractale des courbes intensité-duré-fréquence des précipitations, C.R.A.S., Paris, 325, 323-326,

[24] Marsan, D., SChertzer, D., Lovejoy, S. (1996) Causal space-time multifractal processes: predictability and forecasting of rain fields, Journal of Geophysical Research, 101, D21, 26333-26346.

[25] HURST, H.E. (1951) Long-term storage capacity of reservoirs, Trans Am. Soc. Civ. Eng., 116, 770-808.

[26] TURCOTTE, D.L., GREENE, L. (1993) A scale-invariant approach to flood frequency analysis, Stochastic Hydrol. Hydraul., 7, 33-40.

[27] Tessier, Y., Lovejoy, S., Hubert, P., Schertzer, D., Pecknold, S (1996) Multifractal analysis and modeling of rainfall and river flows and scaling, causal transfer functions, Journal of Geophysical Research, 101. D21, 26427-26440.

[28] Pandey, G., LOVEjoy,S., SChertzer. D. (1998) Multifractal analysis of daily river flows including extremes for basin of five to two million square kilometres, one day to 75 years, Journal of Hydrology, 208, 62 81 .

[29] Water Resources Council (1967) A Uniform Technique for Determining Flow Frequencies, Water Resources Council Bulletin, 15. 